\title{
Single atom spectroscopy: decreased scattering delocalization at high energy losses, effects of atomic movement and x-ray fluorescence yield
}

\author{
Luiz. H. G. Tizei, Yoko Iizumi, Toshiya Okazaki, Kazu Suenaga \\ Nanotube Research Center, National Institute of Advanced Industrial Science and \\ Technology (AIST), Tsukuba 305-8565, Japan \\ Ryo Nakanishi, Ryo Kitaura, Hisanori Shinohara \\ Department of Chemistry, Nagoya University, Nagoya 468-8602, Japan
}

\begin{abstract}
Single atom localization and identification is crucial in understanding effects which depend on the specific local environment of atoms. In advanced nanometer scale materials, the characteristics of invidual atoms may play an important role. Here, we describe spectroscopic experiments (electron energy loss spectroscopy, EELS, and Energy Dispersed X-ray spectroscopy, EDX) using a low voltage transmisson electron microscope designed towards single atom analysis. For EELS, we discuss the advantages of using lower primary electron energy (30 $\mathrm{keV}$ and $60 \mathrm{keV}$ ) and higher energy losses (above $800 \mathrm{eV}$ ). The effect of atomic movement is considered. Finally, we discuss the possibility of using atomically resolved EELS and EDX data to measure the fluorescence yield for x-ray emission.
\end{abstract}

Keywords: EELS, EDX, STEM, Single atoms

\section{Introduction}

Single atom identification has been a recurring theme in condensed matter physics and material science [? ? ? ? ]. The ability to control single atoms or point defects increases the need to correctly identify the local crystal and 5 electronic structures of these entities. Quasi-elastic electron scattering imaging 
(annular dark field images, ADF) using a focused electron probe in a scanning electron microscope (STEM) allows the localization of single heavy atoms inside a lighter matrix, such as Sb atoms in Si [? ]. Scattered intensity is proportional to the atomic number, $Z,[?$ ? ? ] allowing atoms identification in propitious cases [ $\left[\begin{array}{lllllll} & ? & ? & ?\end{array}\right]$. Electron energy-loss spectroscopy (EELS) [? $\left.\quad ? \quad\right]$ and energy dispersed X-ray spectroscopy (EDX) [? ] are techniques that allow the identification of atomic species in a material based on their spectroscopic signatures. EELS may also give information about the local crystal and electronic structures of the probed species [? ? ]. Single atom identification using EELS has been a reality for over a decade [? ? ]. EDX single-atom identification has also been recently demonstrated [? ? ].

In EELS experiments, delocalization effects limit the spatial resolution attainable for a given energy loss and electron probe primary energy, $E[?$ ? ? ]. The benefits of using lower electron probe energy are well known and have 20 been demonstrated [? ? ? ? ]. Moreover, the use of higher energy losses decrease delocalization. For example, for lanthanide atoms $N$-edge absorption edges (between $100 \mathrm{eV}$ and $250 \mathrm{eV}$ ) are typically used for identification. Spatially resolved spectroscopy using those edges results in spatial resolution of the order of $3 \AA$ ref. [? ]. The use higher energy losses ( $M$ edges, above $800 \mathrm{eV}$ ) 25 results in increased spatial resolution [? ? ? ? ? ? ].

Here we report spectroscopic experiments (EELS and EDX) performed on lanthanide atoms trapped inside $\mathrm{C}_{82}$ fullerenes loaded in carbon nanotubes (peapods of La, Ce and Er), molecules known as metalofullerenes [? ] and in europium $(\mathrm{Eu})$ chains confined inside carbon nanotubes [? ]. We describe the benefits of lowering the energy of the probe electrons (Sections 3.1 and 3.2) for EELS experiments. This is followed by a description of the effects of atomic movement (Section 3.3) in EELS data acquisition. Specifically, a fortuitous benefit of delocalization is analyzed (Section 3.3). Parallel EELS and EDX measurement from single atoms is described in Section 3.4. These experiments have allowed an estimation of the fluorescence coefficient for the $M$ edge of Eu atoms. Finally our conclusions are stated in Section 4 . 


\section{Methods}

High spatial resolution EELS experiments at $30 \mathrm{keV}$ and $60 \mathrm{keV}$ (electron beam primary energy) have been performed on a JEOL 2100F microscope

40 equipped with JEOL Delta aberration correctors (dodecapole type) and cold field emission gun. A Gatan Quantum spectrometer modified for low energy operation has been used. The typical electron beam current at $30 \mathrm{keV}$ and $60 \mathrm{keV}$ was of the order of $20 \mathrm{pA}$. The probe convergence semi-angle was 40 mrad and $48 \mathrm{mrad}$ at $30 \mathrm{keV}$ and $60 \mathrm{keV}$, respectively. ADF inner detection

45 semi-angle was $100 \mathrm{mrad}$ at $30 \mathrm{keV}$ and $60 \mathrm{keV}$, respectively. EELS collection semi-angle was $63 \mathrm{mrad}$ and 59 at $30 \mathrm{keV}$ and $60 \mathrm{keV}$, respectively. EELS exposure times of the order of 10-50 ms for $N$ edges and 50-100 ms for $M$ edges. In the EELS filtered maps shown, background contribution was estimated using a power law and two $50 \mathrm{eV}(15 \mathrm{eV})$ wide windows while the edges signal were measured in a $50 \mathrm{eV}(15 \mathrm{eV})$ wide window for the $M(N)$ edges.

Parallel EELS and EDX experiments at $60 \mathrm{keV}$ have been performed in an ARM200F equipped with a Shottky field emission gun and CESCOR CEOS aberration corrector (hexapole type). A Gatan Enfina spectrometer has been used for EELS. For EDS experiments a Silicon Drift Detector (SSD) with 100 $\mathrm{mm}^{2}$ active detection area (Centurio, JEOL) has been used. The detection solid angle, defined by the device geometry in the microscope, is $0.80 \mathrm{sr}$. The typical probe current was $70 \mathrm{pA}$.

Two kinds of samples have been used. The first one was formed of single Lanthanide atoms trapped inside $\mathrm{C}_{82}$ fullerenes. Three different types of 60 atoms have been used: lanthanum ( $\mathrm{La}, Z=57)$, cerium $(\mathrm{Ce}, Z=58)$ and erbium (Er, $Z=68$ ). The $\mathrm{C}_{82}$ molecules containing single atoms have been co-doped into single-wall carbon nanotubes (SWNT) [? ]. The second sample used was composed of europium $(\mathrm{Eu}, Z=63)$ double atomic chains confined inside double-wall carbon nanotubes (DWCNT)[? ].

For La and Ce, $N$ and $M$ EELS measurements at $60 \mathrm{keV}$ can be performed in parallel. For the other atoms and for the experiments at $30 \mathrm{keV}, N$ and 
$M$ measurements have been performed in series. The limitation for these experiments is the minimum dispersion possible of the energy spectrometer (300 $\mathrm{meV} /$ channel and $500 \mathrm{meV} /$ channel at $30 \mathrm{keV}$ and $60 \mathrm{keV}$, respectively)

Where noted, the following kernel was used to smooth images: $\{1,2,1 ; 2,4,2 ; 1,2,1\}$.

\section{Results}

\subsection{Single atom spectroscopy using $60 \mathrm{keV}$ and $30 \mathrm{keV}$ electrons}

EELS has been performed at $60 \mathrm{keV}$ (Fig. 1) and $30 \mathrm{keV}$ (Fig. 2) on single lanthanide atoms ( $\mathrm{La}, \mathrm{Ce}$ and $\mathrm{Er}$ ) confined in $\mathrm{C}_{82}$ molecules which have been co-doped in SWNT. An ADF image of the resulting structure is shown in Fig. 1a, where the heavy atoms appear as bright spots and the supporting carbon structures appear grey. Single atom spectroscopy can be performed either using the $N_{4,5}$ or the $M_{4,5}$ edges for lanthanides. Single atomic column $N_{4,5}$ and $M_{4,5}$ comparisons have been performed in the past [? ? ? . Here, we show $N_{4,5}$ and the $M_{4,5}$ in one of the thinnest possible samples, where electron propagation effects plays a minor role.

The $N_{4,5}$ edges occur due to energy absorption events from electrons in the $4 \mathrm{~d}$ levels and appear in the $100-200 \mathrm{eV}$ energy range. Their energy values for the atoms used in the experiments reported here are: $\mathrm{La} N=117 \mathrm{eV}, \mathrm{Ce}$ ${ }_{85} \quad N=121 \mathrm{eV}, \mathrm{Eu} N=133 \mathrm{eV}$ and $\operatorname{Er} N=164 \mathrm{eV}$ ref. [? ]. These are delayed edges, with a spreadout energy-threshold caused by the orbital momentum of the initial state [? ]. For this reason the determination of the energy threshold may be hindered by the background and noise. The $M_{4,5}$ edges have a smaller delocalization due to their higher energy thresholds, appearing between $800 \mathrm{eV}$ and $1600 \mathrm{eV}$. These edges occur due to transitions of electrons in the $3 \mathrm{~d}$ levels. One important advantage of $M_{4,5}$ edges is the presence of characteristic sharp peaks known as white lines [? ], which appear due to $3 \mathrm{~d} \rightarrow 4 \mathrm{f}$ transitions. In lanthanides, with the exception of Lutetium, a high density of unoccupied $4 \mathrm{f}$ levels exist, explaining the high probability of losses observed [? ]. The energy 
Ce $M=881 \mathrm{eV}, \mathrm{Eu} M=1128.5 \mathrm{eV}$ and $\operatorname{Er} M=1410 \mathrm{eV}[?]$.

The benefits of performing single atom detection using these higher energy losses are greater spatial resolution (decrease delocalization, as described in Section 3.2) and higher energy separation between signals of different atoms preventing edges superposition (Fig. 1b). Energy shifts of the white lines allow the distinction of difference valence state of lanthanides [? ], even at atomic resolution [? ]. For these reasons, whenever possible, $M_{4,5}$ should be preferred.

Because of detectors limitations, reduced total electron probe current in a subnanometer probe and to minimize the total electron dose, $N_{4,5}$ edges have speeds (exposure times of the order of $50 \mathrm{~ms}$ or faster)

Fig. 11-e shows a typical result for La, Ce and Er identification at $60 \mathrm{keV}$ using $M_{4,5}$ edges (blue, green and red, respectively in the color map) . In Fig. 1b, spectra of La, Ce and Er are shown (summed over 9 pixels for La and Ce and 16 pixels for Er).

It is known that lanthanide atoms are free to move inside $\mathrm{C}_{82}$. This movement is increased due to the influence of the $60 \mathrm{keV}$ electron beam. For this reason, extra atoms are seen (e. i. atoms which were not present before spectrum image acquisition, Fig. 15) in the ADF image acquired in parallel to the 1]-e. These extra atoms could have appeared due to movement of the same atom inside of the $\mathrm{C}_{82}$. However, the EELS signal clearly shows them to be different: the first pair is Ce-La and the second one Ce-Er. These atoms came from elsewhere, demonstrating the necessity of spectroscopy to identify atoms ${ }_{125}$ (as their atomic numbers are too close to prevent discernment by ADF con- 
(a)

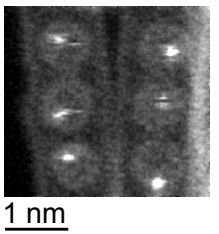

(b)

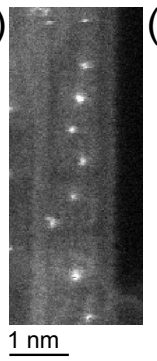

(e)

(f)
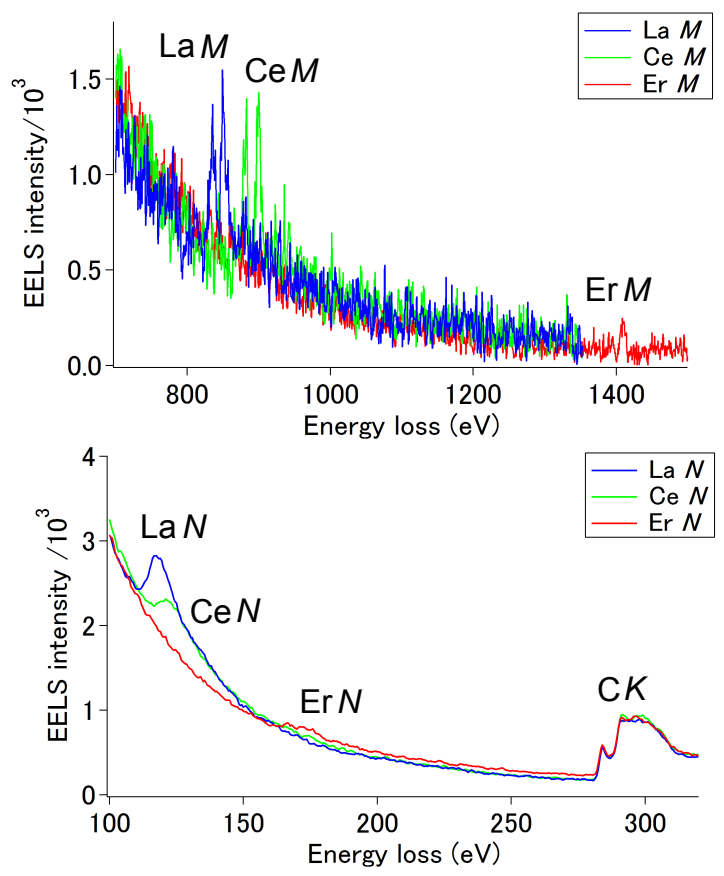

Figure 1: Single atom spectroscopy at $60 \mathrm{keV}$. (a) Typical ADF images showing the lanthanide atoms (bright spots) confined inside $\mathrm{C}_{82}$ fullerenes, which were co-doped in SWNT. (b-d) 2D EELS mapping of the $M$ edges shown in (e). (b) ADF image before EELS acquisition and (c) is the ADF during EELS acquisition. (d) Color-coded image showing the position single La (blue), Ce (green) and Er (red). Two pairs of atoms described in the text are pointed by arrows in (d). The acquisition time per pixel was $50 \mathrm{~ms}$ for this map. (c) and (d) have been smoothed. (e-f) Single atom spectra (marked by white squares in e)) for the $M$ and $N$ edges,respectively, of La (blue), Ce (green) and Er (red). The total exposure times for the $M$ spectra were $450 \mathrm{~ms}$ for La and Ce and $800 \mathrm{~ms}$ for Er. 
trast). Furthermore, this shows that these are not multiple images of the same atom oscillating, which can also be observed [? ], as described in Section 3.3

Substantial damage can occur to fullerenes under irradiation from a $60 \mathrm{keV}$ or above electron beam [? ? , even though this energy is below the knock-on threshold for Carbon. Evidently, a smaller influence from the electron beam is desirable. Electron microscopy experiments at $20 \mathrm{keV} \mathrm{[?} \mathrm{],} 30 \mathrm{keV} \mathrm{[?} \mathrm{]} \mathrm{and}$ $40 \mathrm{keV}[?]$ creates significantly less damage and atomic natural movement (not induced by the electron beam) may be observed in specific situations. This decrease in damage is true for system in which knock-on plays an important role.

Indeed, we have observed significantly less damage for the fullerene sample at $30 \mathrm{keV}$. However, atomic movement cannot be suppressed. In Fig. 2 one example of single atom identification using the $N_{4,5}$ at $30 \mathrm{keV}$ is shown. The $\mathrm{C}_{82}$ molecules show their original shape even after several 2D maps acquisition. In fact, a double image of an atom can be seen even in the $2 \mathrm{D}$ map (arrow in Fig. 2b). The two images are separated by $0.2 \mathrm{~nm}$. However, an accurate measurement of the distance between the stable positions is not possible as it depends on the angle between a line joining the atomic pair and the electron beam (which we cannot estimate). The reason behind this double image is either the movement of the atom inside the $\mathrm{C}_{82}$ molecule, or a movement of the system as a whole (the $\mathrm{C}_{82}$ and the atom). Usually, such states are observed in images (acquisition time of the order of tens of $\mu \mathrm{s}$ ) but not in the $2 \mathrm{D}$ chemical maps at $60 \mathrm{keV}$ (Section 3.3). The exposure time for this $2 \mathrm{D}$ chemical map was $10 \mathrm{~ms}$ per pixel. Fast acquisition was possible due to the higher inelastic cross section at lower energy-loss and electron primary energy.

In the same 2D EELS mapping the carbon $K$ edge (threshold around $284 \mathrm{eV}$ ) could be acquired in parallel. A map of this edge distinctly shows the contrast from the fullerenes shell (Fig. 2d). A hindrance to 2D chemical mapping of many atomic species at $30 \mathrm{keV}$ is the limited energy range available to EELS.

155 This happens due to the minimum natural dispersion of the energy spectrometer used. For this reason $M_{4,5}$ mapping of La, Ce and Er is not possible in parallel. 
(a)

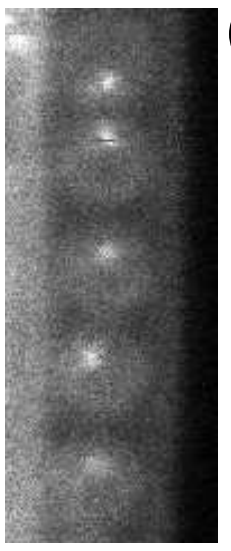

$1 \mathrm{~nm}$ (b)

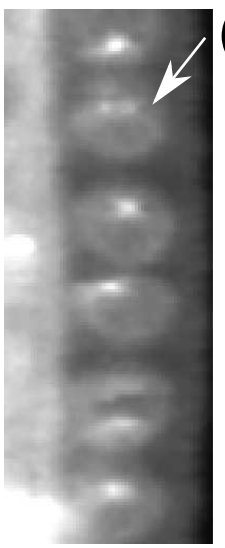

$1 \mathrm{~nm}$ (c)

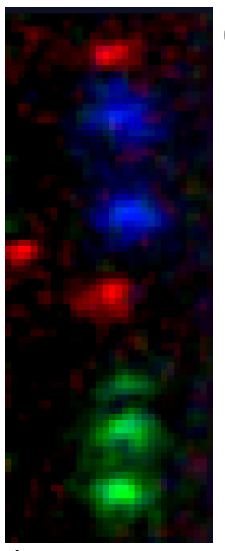

$1 \mathrm{~nm}$ (d)

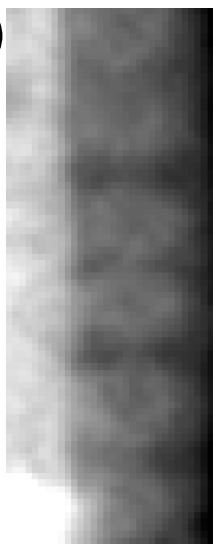

$1 \mathrm{~nm}$

(e)

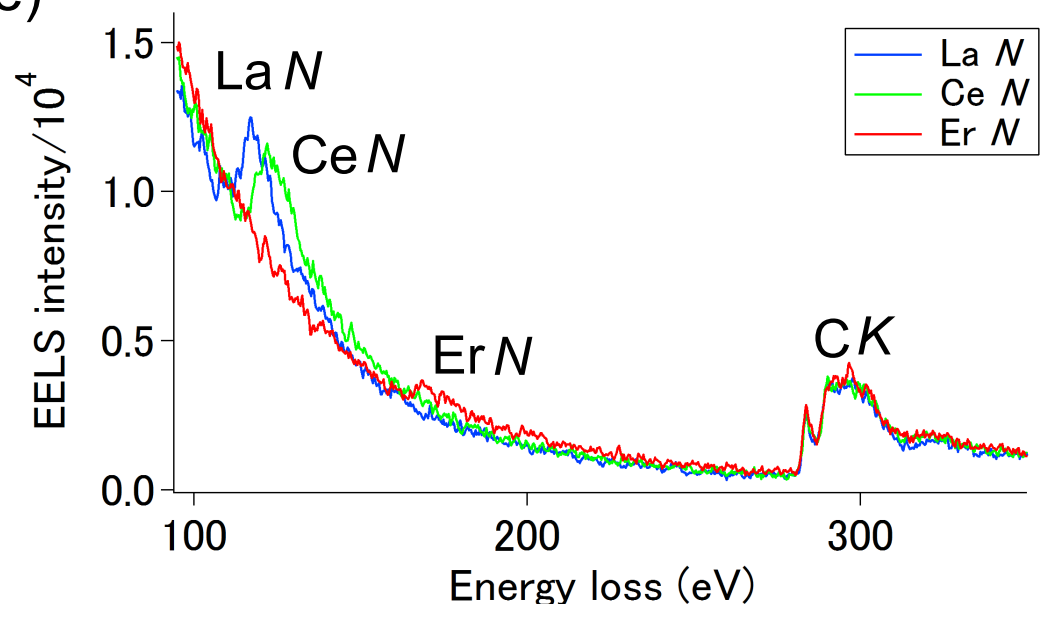

Figure 2: Single atom spectroscopy at $30 \mathrm{keV}$. ADF images acquired before (a) and during (b) the 2D EELS map acquisition. (c) Color-coded La (blue), Ce (green) and Er (red) N edge maps. The exposure time per pixel was $10 \mathrm{~ms}$. (d) C K edge map, distinctly showing the contrast from the fullerenes. e) spectra from La (blue), Ce (green) and Er (red) N edges averaged over 25 pixels (total acquisition time $250 \mathrm{~ms}$ ). (b-d) have been smoothed 
However, this is feasible in series of measurements. Finally, as explained in detail in Section 3.3, atomic movement happens continuously in this system. This greatly limits our capabilities for 2D mapping, as it poses an upper bound on the typical exposure time before the system changes. Typically, we have used acquisition times of the order of $50 \mathrm{~ms}$ to mitigate this effect.

\subsection{Decrease in inelastic scattering delocalization: effect of energy loss and and primary electron energy}

The spatial resolution of 2D EELS map is fundamentally limited by the delocalization in inelastic scattering [? ? ? ]. Delocalization occurs because of the non-zero probability of energy loss at impact parameter $b$. Essentially, the probability of energy loss decreases as $\exp \left(-2 b / b_{\max }\right)$ for $b>b_{\max }$ and as $1 / b_{\max }^{2}$ for $b<b_{\max }$, ref. [? ]. $b_{\max }$ is given by:

$$
b_{\max }=\frac{2 \pi \hbar v}{\Delta E},
$$

where $b_{\max }$ gives the spatial extent of the delocalization and the limit for the resolution in 2D mapping. This quantity depends on the energy loss $(\Delta E)$ and incident electron primary energy ( $E$ through $v$, the electron speed, in equation 1). However, comparison with experiments shows that such reasoning leads to predictions larger than the observed values. The reason for this is the extended angular distribution of inelastic scattering [? ? ]. Egerton [? ? ] has predicted that the image of an atom should have a width at $50 \%$ intensity equal to:

$$
d_{e}(\Delta E)=\frac{\lambda}{2}\left(\frac{2 E}{\Delta E}\right)^{\frac{3}{4}} .
$$

The effect of delocalization can be clearly seen in the Fig. 3a-d, which shows the spatial distribution of the inelastic scattered intensity at two energy losses, the $N$ edge at $\Delta E=125 \mathrm{eV}$, the $M$ edge at $\Delta E=881 \mathrm{eV}$ and their profiles at $E=60 \mathrm{keV}$, for two Ce atoms. This difference can be quantified from the position at which the intensity has dropped to $50 \%$ in the loss maps. This position can be measured from profiles of the ADF and EELS maps (Fig. 3d). 
At $60 \mathrm{keV}$ the measured values are $(0.15 \pm 0.02) \mathrm{nm},(0.32 \pm 0.02) \mathrm{nm}$ and $(0.20 \pm 0.02) \mathrm{nm}$, for the $\mathrm{ADF}, \Delta E=125 \mathrm{eV}$ and $\Delta E=881 \mathrm{eV}$, respectively. From equation 2 the theoretical values for $60 \mathrm{keV}$ are $d_{e}(125 \mathrm{eV})=0.42 \mathrm{~nm}$ and $d_{e}(881 \mathrm{eV})=0.10 \mathrm{~nm}$. The measured value for $d_{e}(125 \mathrm{eV})$ is significantly smaller than the theoretical one. We do not have a solid interpretation for this difference, but it might be due to convergence and collection angles effects which are not considered in our simple calculation. The $d_{e}(881 \mathrm{eV})$ is larger than predicted, which occurs because of the finite electron probe size. The decrease in the $\Delta E=881 \mathrm{eV} 2 \mathrm{D}$ map is sufficient to discern the effect of a jump of the Ce atom closer to the bottom of the image (pointed by arrows in Fig. 3a and 3.). This jump appears only as a blur in the $\Delta E=125 \mathrm{eV}$ image. Such jumps are inevitable, as described in Section 3.3 .

For $30 \mathrm{keV}$ electrons delocalization should be smaller, as seen in equation 2. The theoretical values are $d_{e}(125 \mathrm{eV})=0.36 \mathrm{~nm}$ and $d_{e}(881 \mathrm{eV})=0.08 \mathrm{~nm}$. The $\Delta E=125 \mathrm{eV}$ and $\Delta E=881 \mathrm{eV}$ could not be measured in parallel and have been measured sequentially. The delocalization values at $50 \%$ intensity are: $(0.36 \pm 0.02) \mathrm{nm},(0.30 \pm 0.04) \mathrm{nm},(0.34 \pm 0.02) \mathrm{nm}$ and $(0.38 \pm 0.02) \mathrm{nm}$, case.

\subsection{Atomic movement in metal fullerenes: effects of delocalization}

It is known that a lanthanide atom can have more than one stable equivalent position inside a $\mathrm{C}_{82}$ molecule, in absence of external influences [? ]. At room temperature, translation between these positions can be thermally activated. Translation occurs in time scales much shorter than typical exposure times in 


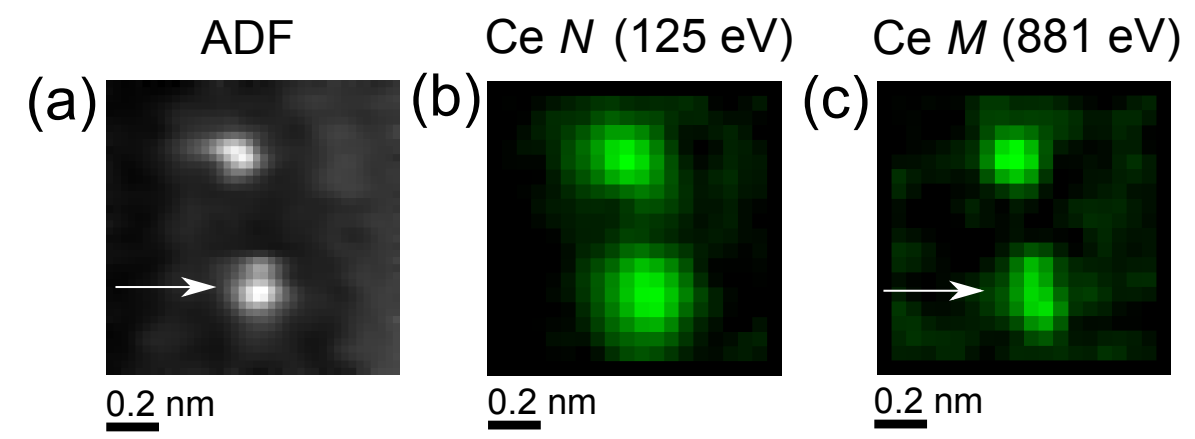

(d)

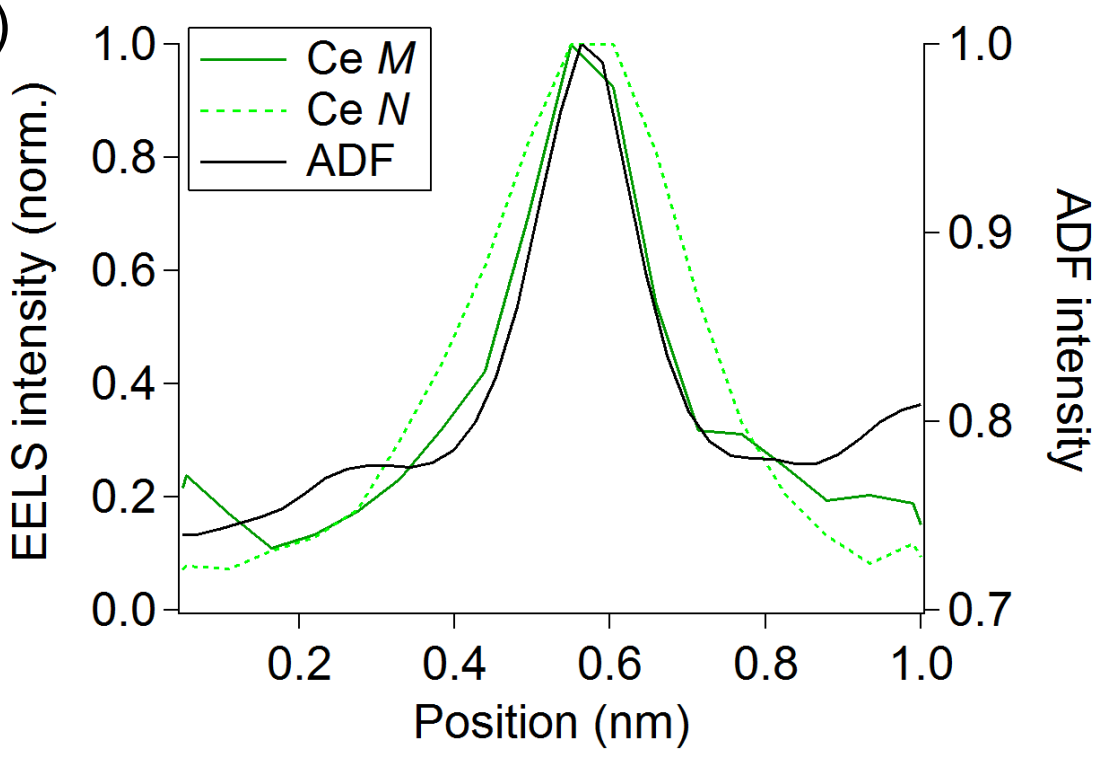

Figure 3: (a-c) ADF and chemical maps at two different energy losses (125 eV and $881 \mathrm{eV}$ ) for two Ce atoms. The EELS maps have been made close the threshold energy for the $N$ and $M$ edges of Ce. Note that the spatial resolution in (c) is similar to that in (a). The broadening in (b) happens due to inelastic scattering delocalization. (d) comparison of the ADF, Ce $N$ and Ce $M$ signals at $60 \mathrm{keV}$. The difference in delocalization at two energy losses is clearly seen in (d). (a-c) have been smoothed. 
ADF imaging (tens of $\mu \mathrm{s}$ ). Hence, a hopping atom appears as multiple atoms (multiple images of the same atom) with reduced total intensity, as shown in Fig. 4a-d. In Fig. 和 two pairs of images with half intensity when compared to a stable atom are seen (profiles in Figure 4c). In Figure 4b a pair of images of a moving atom is seen close to a real pair of stable atoms (profiles in Fig. 4 $\mathrm{d}$ ). Multiple images of the same atom due to this effect have been reported before [? ]. We should note that the transition time must be much shorter than scan time of a few pixels. If the oscillation time was much longer alternating darker (no atom) and brighter pixels should be seen. Indeed such behavior can also be found in this system. One example is shown in Fig. $2 \mathrm{~b}$ (lower part), where the center portion of a $\mathrm{C}_{82}$ appears significantly darker than its upper and lower portions.

Transitions between hopping and a single stable position are routinely observed in this material. This occurs probably due to geometric changes of the $\mathrm{C}_{82}$ molecule induced by the electron beam.

In our experiments at $30 \mathrm{keV}$ we have been able to measure the spectroscopic signature of hopping atoms. An example is shown in Fig. 2 b, where a double image of an atom is seen. EELS showed that the atom was a Ce (not two lighter atoms, for example), confirming that the decrease in intensity was due to translation.

The EELS signal can also be used to detect and identify atoms which cannot be imaged in ADF images due to movement. In Fig. 使 we show an ADF image acquired prior to 2D EELS mapping in a region containing two atoms (in the marked rectangle). During mapping one of the atoms cannot be seen in the ADF image acquired and it seems to have left the $\mathrm{C}_{82}$ (Figure 4f). However, both atoms can be detected in the 2D $N_{4,5}$ EELS map (Fig. 4 4 ). The upper one is a Ce and the lower one a La (confirmed by a successive $M_{4,5}$ EELS map).

Although more spread out, the total intensity of the integrated spectrum of the Ce and the La atoms is not significantly different. However, the "local" intensity is indeed different: for a given region the signal for the Ce atom is twice as large as for the La atom. This decrease in intensity is expected when compar- 
(a)

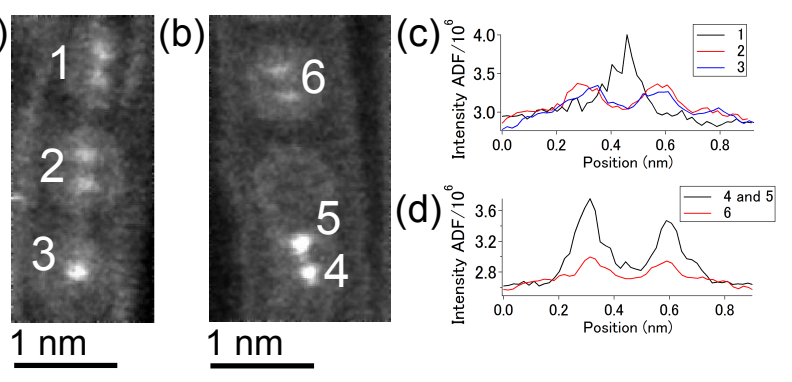

(e)

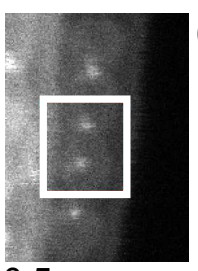

$\underline{0.5 \mathrm{~nm}}$

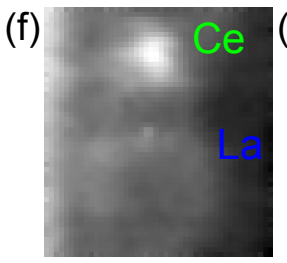

$\underline{0.5 \mathrm{~nm}}$

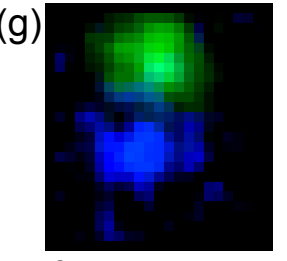

$\underline{0.5 \mathrm{~nm}}$

(h)

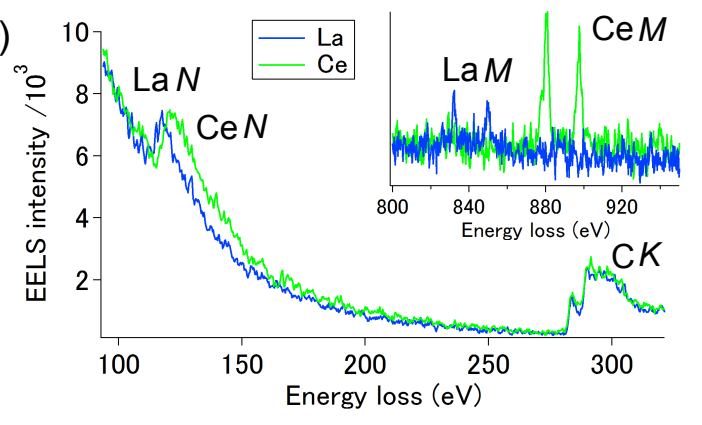

Figure 4: (a-b) ADF images comparing atoms with two stable positions (1,2 and 6) with those with one stable position (3,4 and 5). The distinction can be made based on the ADF intensity. This can be seen in more detail in the profiles shown in (c-d). (e-g) 2D EELS map a Ce and La atom. During the map acquisition the La atom cannot be seen in the ADF image, probably because it is moving between many stable positions. However, the atom can clearly be seen in the EELS map (g). (h) N and M edges of La (blue) and Ce (green) from this EELS map confirming the atoms are indeed Ce and La. (f-g) have been smoothed. 
ing a measurement with small impact parameter and in aloof geometry. This observation is probably explained by delocalization (described in Section 3.2). That is, even during the period when the atom was away from the electron beam (larger impact parameter) the probability for inelastic scattering (EELS signal) was still high due to delocalization. However, the same is not true for elastic scattering (ADF image). For this reason the atom can be seen in the EELS map but not on the ADF map. This is a fortuitous benefit of delocalization in spectroscopic measurement.

Finally, this natural atomic movement is a great hindrance for atomically resolved spectroscopy in general. For our EELS experiments, our maximum exposure time has been typically limited by the stability of the atom in the peapod during imaging. Note that the movement is not induced only by the 255 electron beam and cannot be avoided in this case. For the lanthanide atoms inside peapods this translates to roughly $50 \mathrm{~ms}$ or $100 \mathrm{~ms}$ per pixel. Still in this time range, atomic jumps were routely observed. Atomic movement is one of the main limitations for single atom EDX spectroscopy [? ? ] (Section 3.4), where exposure times are much longer due to poor collection efficiency.

\subsection{Single atom $X$-ray spectroscopy}

EDX is a well-adapted tool to study materials containing heavier atoms. The flux of X-ray photons from a given excited atom depends on the fluorescence yield, $\omega$, of a given transition. $\omega$ is the ratio of number of X-ray emission events to the number of created core-holes (or vacancies) for a specific transition. Typically, fluorescence yield measurements are performed using X-ray fluorescence spectroscopy [? ? ], where an energy-filtered X-ray source can be used to selectively create core-holes in a shell of the probed atom.

Here we discuss the possibility, that has already been suggested by Lovejoy et al [? ], of performing similar measurements in single atoms using EELS and EDX to probe the excitation and decay events, respectively. One difficulty for such experiment is the choice of the atom to be probed. We have chosen Eu because its $M_{4,5}$ edge occurs around $1100 \mathrm{eV}$. This energy is sufficiently 
high to avoid absorption effects on the vacuum-window of the EDX detector and sufficiently low to allow EELS measurements (non-negligible inelastic cross

In Fig. 5] an experiment at $60 \mathrm{keV}$ with parallel EELS and EDX acquisition on Eu chains is shown (Fig. 5a-b). The acquisition time to get a statistically significant EDX signal is about $500 \mathrm{~ms}$, with a probe current of $70 \mathrm{pA}$ (the current was limited by the requirement to have a sufficiently small probe to distinguish the atoms separated by $3.5 \AA$ ). However, even with these long exposure times 2D mapping of the EDX $L$ and $M$ peaks signal is poor (Fig. 5 r-d shows the integrated Eu $M$ and $L$ signals). An average EDX spectrum on the position of a single Eu atom (12.5 s acquisition time, marked by a red square in Fig. 5b-d) of the $M$ (Fig. 55) and the $L$ peaks (Fig. 5f) are shown. A background signal is shown for comparison in green (integrated on the green rectangle in Fig. 5d). A global average spectrum (198 s acquisition time) of this section of the Eu chain is shown in Fig. $5 \mathrm{~g}$, where the $M(\alpha, \beta$ and $\gamma)$ and $\mathrm{L}(1, \alpha, \beta$ and $\gamma)$ peaks are seen.

Total X-ray emission and absorption events have been measured on a region where a single atom can be identified in the ADF image (red square on Fig. 5(b-d)). The absorption signal has been corrected for the CCD efficiency and the finite convergence and collection angles. The EDX map shown (5)d) does not allow single atom identification.

The X-ray signal was corrected by a geometric factor due to the detector's solid angle. Moreover, the number of $M$ transitions depends on the number of $M$ shell core-holes. After these corrections our measurements are: $E D X_{M}=$ $(550 \pm 90)$ photons, $E D X_{L}=(700 \pm 100)$ photons and $E E L S_{M}(100 e V)=$ $(1840 \pm 20) M$ shell core-holes created. Due to the finite energy integration window $(100 \mathrm{eV})$ the number $M$ shell core-holes created by absorption events has to be corrected. We have estimated the number of missed events using power laws (this is largest source of uncertainty).

The majority of $M$ shell core-holes are created by the primary electron beam. These represent direct absportion events, which can be measured from the EELS 

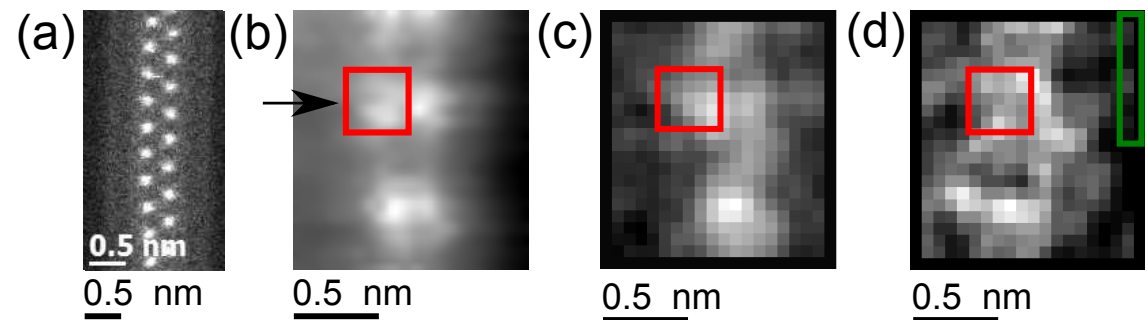

(e)

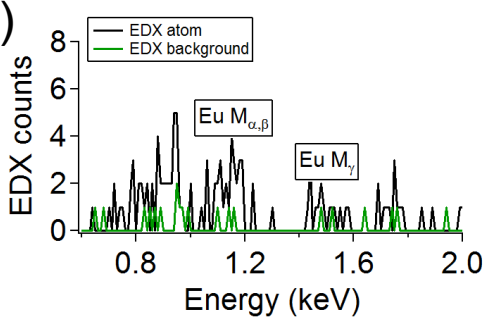

$\underline{0.5 \mathrm{~nm}}$

$0.5 \mathrm{~nm}$

(f)

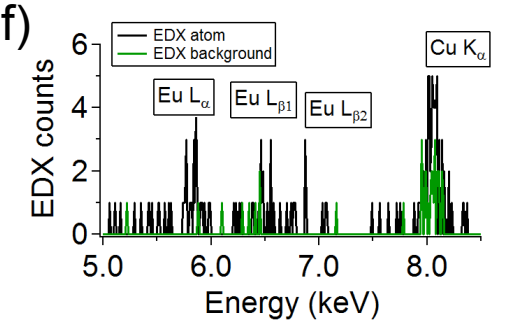

(g)

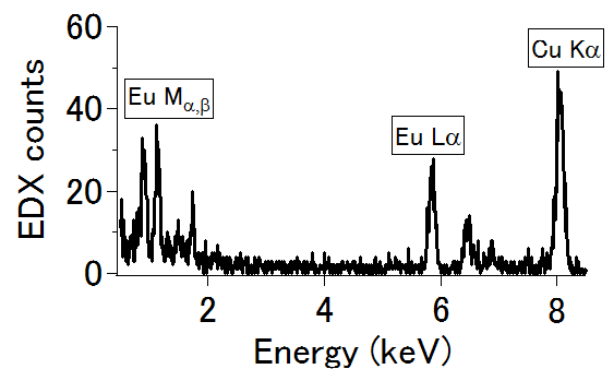

Figure 5: (a) Typical ADF image of a Eu atomic chain inside a DWCT. (b-d) ADF image acquired during 2D mapping, EELS Eu M edge map and EDX Eu M peak map. The acquisition time per pixel was $500 \mathrm{~ms}$. (e-f) $M$ and $L$ lines EDX spectrum for one atom integrated integrated on the red square in (b-d). The total integration time was $12.5 \mathrm{~s}$. The background was integrated on the green rectangle, with the same total exposure. (g) Global EDX spectrum (198 s integration time) showing the Eu M and L peaks. Cu from the sample grid and Si from the detector can also be seen. (b-d) have been smoothed. Not all lines are identified in the spectra. 
signal. However, $L$ transitions (radiative and non-radiative) also create $\mathrm{M}$ shell

core-holes[? ]. These have been estimated using the $L$ peak X-ray intensity and calculations for the average Auger $\left(a_{L}\right)$ and fluorescence yield $\left(\omega_{L}\right)$ coefficients for the $L$ peak (4000 extra core-holes). Coster-Kronig $M$ sub-shell transitions were not considered, as the signal from all the $M$ transitions has been integrated.

With all these correction the number of core-holes lie in the range $E E L S_{M}($ all $)=$ [14100;24400]. The lower bound comes from an $E^{-3}$ and the upper bound from an $E^{-2}$ fit to the Eu EELS $M$ edge. With these numbers the estimated fluorescence yield lies between 0.02 and 0.03 (the theoretical value is 0.0136 ref. [? ]). The large uncertainty stems from the necessity to extrapolate the tail of the EELS $M$ edge.

Our results indicate that 2D mapping of the EDX signal in parallel to EELS acquisition from single atoms can be envisaged with an increase in the current density (a decrease from $0.35 \mathrm{~nm}$ to $0.1 \mathrm{~nm}$ translates to approximately ten times larger current density) and a decrease in the electron beam energy to 30 $\mathrm{keV}$ (increase in absorption cross section).

One of the greatest limitation for these experiments is atomic movement. As described in Section 3.3 this limits the exposure time in peapods experiments to times of the order of $100 \mathrm{~ms}$. For the Eu chains, in ideal situations, this time can be extended to up to $500 \mathrm{~ms}$. This happens because relative movements between atoms are less probable in the chains (there is less free space).

\section{4. Conclusion}

We have demonstrated the current possibilities for single atom detection and mapping using EELS and EDX spectroscopies in metallofullerenes and atomic chains confined inside carbon nanotubes. The benefits of lowering the energy of the electron beam from $60 \mathrm{keV}$ to $30 \mathrm{keV}$ have been explored. Moreover, the effects of the energy loss, $\Delta E$, at two different acceleration voltages has been analyzed. The influence of the motion of atoms inside fullerenes has been considered. This motion, which can be induced by the electron beam, greatly 
limits the maximum exposure time used in 2D mapping. Finally, we have measured the X-ray fluorescence yield of the $\mathrm{Eu} M$ edge by measuring in parallel the absorption and fluorescence signals from individual Eu atoms using EELS and EDX. We concluded that the determination of this quantity is hindered by the large uncertainty in the estimation of the absorption tail in the EELS signal. Also, natural atomic movements are identified as one of the major difficulties in single atom EDX identification.

\section{Acknowledgements}

Yoshiko Niimi, Kayoko Sato are gratefully acknowledged for their assistance of specimen preparations and TEM observations. The work is partially supported by the JST Research Acceleration Programme.

\section{References}

\section{References}

